\title{
Grinding Wheel Monitoring System
}

\author{
Igor Nikiforov \\ Departament of Technology of ma- \\ chinebuilding \\ Pskov state university \\ Pskov, Russia \\ Nikiforov.i.p@mail.ru
}

\author{
Pavel Maltsev \\ Departament of Technology of ma- \\ chinebuilding \\ Pskov state university \\ Pskov, Russia \\ Inertan@gmail.com
}

\author{
Marina Kulakova \\ Departament of Technology of ma- \\ chinebuilding \\ Pskov state university \\ Pskov, Russia \\ Kulakovamarinavas@gmail.com
}

\begin{abstract}
It has been hypothesized that the change in the micro and macro geometry of the grinding wheel can be determined by a non-contact way by supplying jet of air through the nozzle of a pneumatic-electric-contact converter to the surface of the tool during operation. To confirm this hypothesis an experiment was did on a surface grinding machine. Various samples were machined, that led to selfsharpening of the tool. Grinding wheel monitoring system was developed for the experiments. System includes a device that provides the setting of the necessary working gap between the nozzle and the controlled wheel. A justification is proposed for determining the frequency of grinding wheel grinding during operation by accurately accounting for changes in the state of its surface without stopping the machining process.
\end{abstract}

Keywords-grinding wheel, monitoring system, self sharpening.

\section{INTRODUCTION}

The grinding process of various steels is characterized by self-sharpening and salting of the grinding wheel. In addition, the grains can wear out and break out of the bunch.

Self-sharpening is the process of chipping blunt abrasive grains with the emergence of new cutting edges. This process is positive, however, it leads to an intensive decrease in the dimensional accuracy of processing due to a decrease in the tool diameter and a change in the original shape of the wheel.

Salting is the process of filling the space between cutting abrasive grains with slurry. This process is negative, because the tool loses its cutting ability and cutting is replaced by elastic and plastic deformation. This increases the temperature in the cutting zone, burns and other defects appears on the treated surface.

It is important to know the state of the surface of the grinding wheel during processing, in order to obtain accurate geometrical sizes of the part, to prevent the appearance of defects on the surface and identification of the need to dressing of the wheel.

There are some methods to measure the linear wear of the grinding wheels and to monitor the state of its

surface: contact and non-contact, with stopping the grinding process and without stopping. Let's look at main examples.

There is an indirect method to measure the wear of diamond grinding wheels by measuring the cutting force.

The degree of wear of the diamond wheel can be estimated by the level of forces and restore its cutting properties in a timely manner. Many researchers made an experimental determination of value and nature of changes in cutting forces during grinding process by using various measuring tools. Firstly, the cutting force was determined by measuring the power developed by the electric motor during grinding with a wattmeter. The value of the tangential component of the cutting force was determined by calculation. This method is simple, but it does not allow to measure the components of the cutting forces and is not accurate, because it determines the total power, including friction losses in the machine mechanisms. Dynamometric devices can be used to directly measure the component forces. However, this method is valid only when parts are installed in centres [1].

There is a method of contactless determination of the wear of the grinding wheel according to the parameters of the vibration of the machine. The essence of this method is the measurement of the distance between the grinding wheel and the capacitive sensor. Capacity changes with relative fluctuations. Changing the capacitance using a special amplifier is converted into an electrical signal, which is recorded by a recording device. This method almost does not find practical application, because it has low measurement accuracy and it is characterized by the complexity of processing the measurement results and significant errors [5]. Based on the research [2] was found that the wear of the grinding wheel can be determined by the statistical parameters of the signal of vibration acceleration. The appearance of new vibration components in the range of $2000 \ldots 2200 \mathrm{~Hz}$ was revealed from the spectra of vibration accelerations. Obtaining harmonics of vibration accelerations in two directions allow to get rid of 
the noise components in the signal and solve the problem of diagnosing tool wear at any stage of work.

There are several studies on the measurement of wear and the topography of the grinding wheel by examining changes in hydrodynamic pressure during operation. The studies are based on the analysis of the change in pressure of the fluid that was applied between the grinding wheel and the workpiece being processed. To measure the hydrodynamic pressure used several pressure sensors, which are installed directly near the wheel and the workpiece. When the wheel is worn, the gap between it and the sensor increases, which leads to a decrease in pressure. [3]

Also known methods for contactless analysis of the state of the surface of the grinding wheel using the indicator and optical devices, step-by-step photographing and laser projection with 3D-modeling. Most optical methods are based on laser triangulation. The main elements of the installation: a laser diode and a position-sensitive detector with an amplifier and two lenses [4], [6].

\section{Materials AND METHODS}

One of the most promising is the non-contact pneumatic method for measuring grinding wheels. For this purpose, it is proposed to use bellows-type pneumaticelectric-contact converter, the principle of action of which is based on the change in air pressure in the bellows chamber, when the gap between the nozzle and the measured surface changes. In Fig. 1 shows a diagram of bellows pneumatic-electric-contact converter.

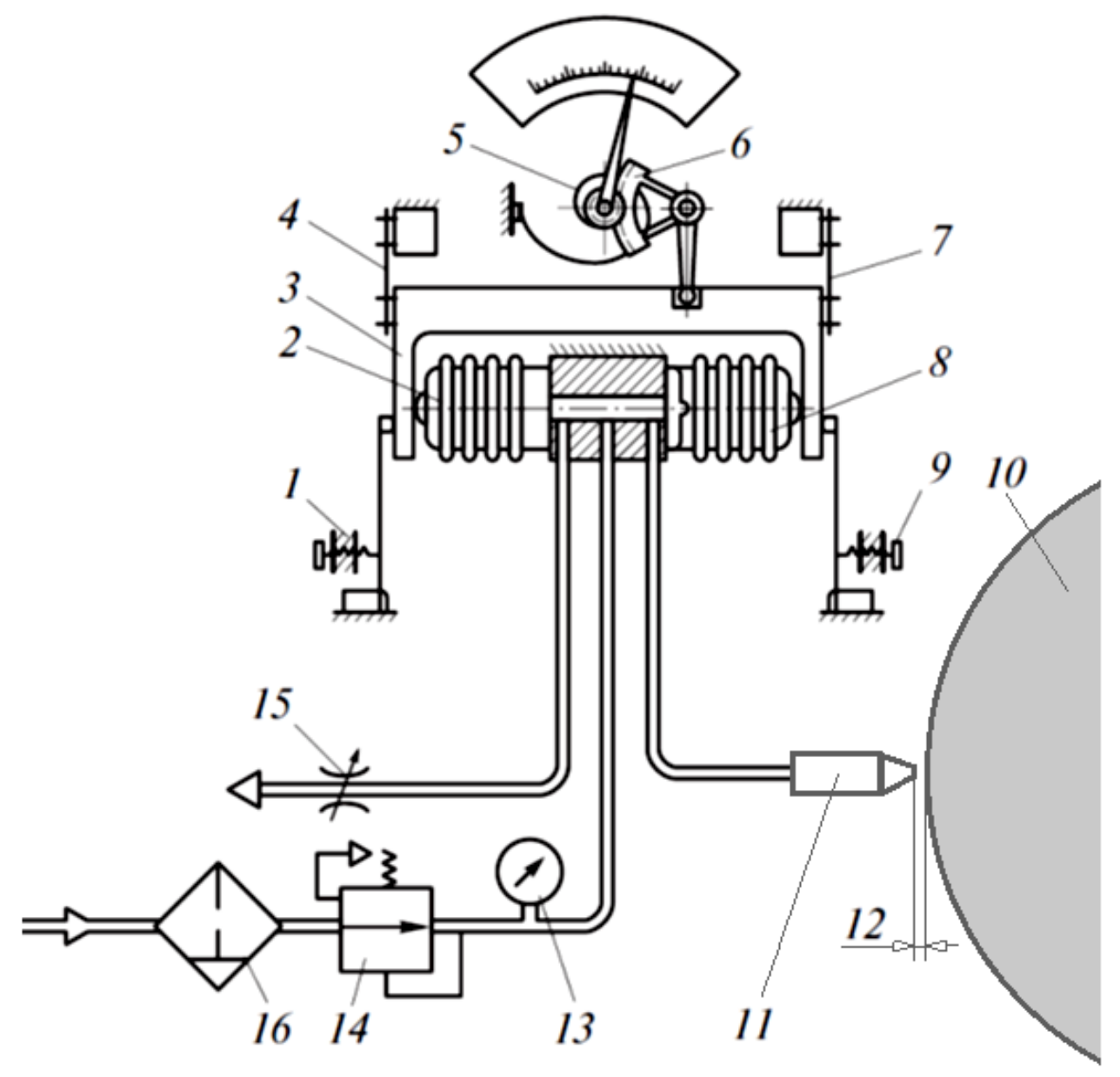

Fig. 1. Scheme of pneumatic-electric-contact convector.

Compressed air is supplied from the compressor through the filter 16 and the reducing valve 14 in the cavity of the left 2 and right 8 bellows (closed brass vessels with corrugated walls). At the same time, the air enters the nozzle 11 and the backpressure valve 15, which is an adjustable air throttle.

The air flow through the valve 15 (at a certain setting) will be constant; the pressure in the left bellows will also remain unchanged. The air flow through the nozzle 11 and the pressure in the right bellows will depend on the actual size of the gap 12 between the surface of the controlled grinding wheel 10 and the end face of the nozzle 11.

When the grinding wheel is worn, the gap 12 between the nozzle 11 and the wheel 10 will increase, the pressure in the right knee of the sensor will decrease, the right bellows will compress, and the left one will stretch. Frame 3 , suspended from the sensor body on flat springs 4 and 7, will displacement to the left and close the adjustable contact 1 . There can be up to six such contacts in commercially available sensors, which close at a different 
value of displacement of the frame 3 in one direction or another. Thus, the whole measurement range can be divided into seven intervals. This allows simplifying the automation of dressing of the grinding wheel.

The use of a differential circuit (with an adjustable backpressure valve) increases the sensitivity of the sensor and reduces requirements for stabilizing the air pressure controlled by a manometer 13 . The converter is equipped with a indicating device: the frame 3 turns the toothed sector 6 , which is connected to the arrow.

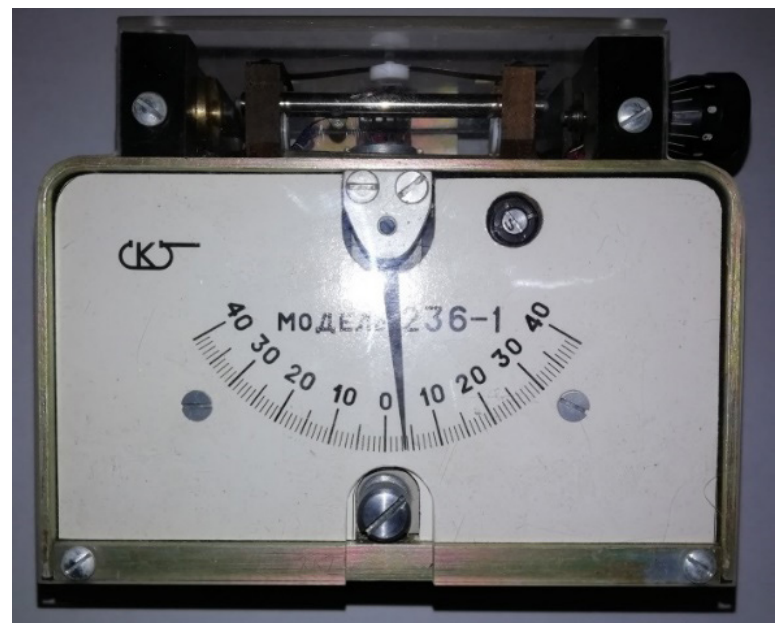

a)
The appearance of the pneumatic-electric-contact converter is shown in Fig. 2, and as a whole grinding wheel monitoring system - in Fig. 3. The system includes the following components: a compressor (not shown in the figure); air preparation unit 1 , in which the air is dried and separated from moisture; choke 2; pressure stabilizer 3; pneumatic-electric-contact converter 4; backpressure valve 5 ; adjusting device 6 with a nozzle (to regulate the size of the gap); grinding wheel 7, connecting pipes.

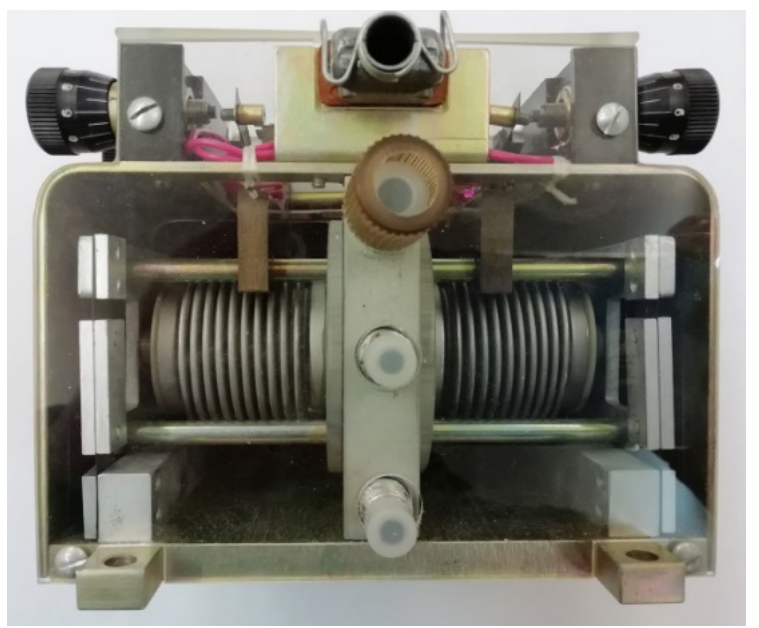

b)

Fig. 2. Appearance of pneumatic-electric-contact converter: $\mathrm{a}$ - front view; $\mathrm{b}$ - rear view.

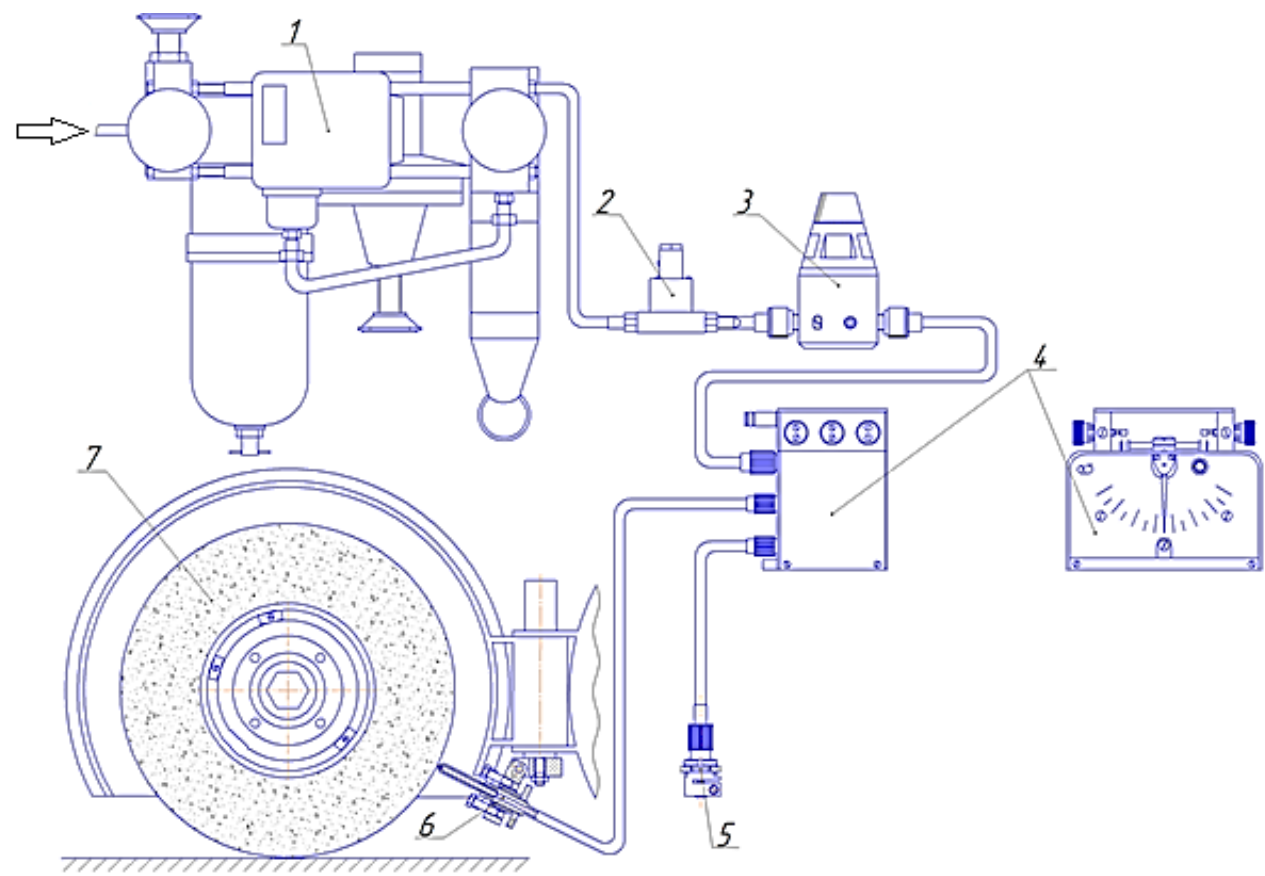

Fig. 3. Grinding wheel monitoring system.

The design of the adjusting device is shown in an adjustment screw 5 in the housing 3 , which fig. 4. The adjusting device consists of a bracket containing a guide screw 6 with a fitting 7 . A 1 mounted on a support 2. A housing 3 is located nozzle 8 is fixed in the guide screw 6 . rotatably around an axis 4 on bracket 1 . There is 


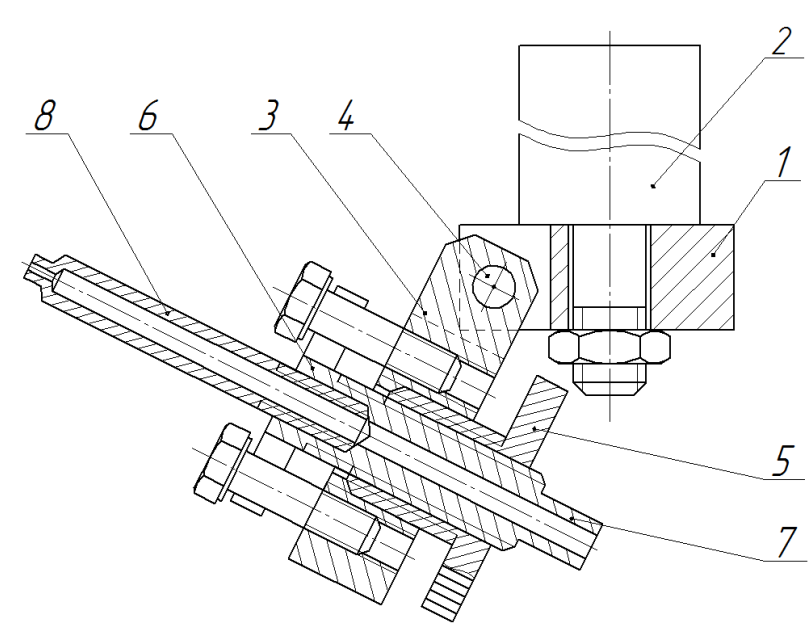

Fig. 4. Adjusting device

The adjusting device operates as follows: support 2 is fixed in the housing protection grinding wheel. The housing 3 with its rotation around the axis 4 positions the axis of the nozzle 8 radially relative to the grinding wheel. The movement of the nozzle 8 to the center of the grinding wheel is carried out by turning the adjusting screw 5 , which causes the guide screw 6 to move. The thread pitch on the surface of the adjusting screw 5 is larger than that of the guide screw 6 , which makes it possible to feed the nozzle 8 smoothly. Air is supplied through fitting 7.

The calibration was performed before the experiment. Wheel dressing was made by a diamond pencil. Depth of dressing was established by a limb of vertical feed of the grinding head of the machine, with an accuracy of 1 micron. The measurement of the radial size of the circle was carried out continuously in the process of dressing. The value of change in the radial size of the circle is equal to the vertical feed. The graduation of the pneumatic-electriccontact convector is $0.2-2$ microns. The required pressure (3,6 MPa) was set using a stabilizer (pos. 3 Fig. 3), which provided a change in the position of the convector arrow by 1 mark on the scale when dressing a wheel by 1 micron.

III. RESULTS AND DISCUSSION

The calibration was performed before the experiment. Wheel dressing was made by a diamond pencil. Depth of dressing was established by a limb of vertical feed of the grinding head of the machine, with an accuracy of 1 micron. The measurement of the radial size of the circle was carried out continuously in the process of dressing. The value of change in the radial size of the circle is equal to the vertical feed. The graduation of the pneumatic-electriccontact convector is $0.2-2$ microns. The required pressure (3,6 MPa) was set using a stabilizer (pos. 3 Fig. 3), which provided a change in the position of the convector arrow by 1 mark on the scale when dressing a wheel by 1 micron. Setting error is $0,003 \%$ on the passport of convector.

Samples made of stainless steel P900 were processed on a surface grinder $3 \mathrm{G} 71$ by the grinding wheel 1 250x20x76 WA F30 L6V 502 made of aluminum oxide white with using the proposed monitoring system. The cutting conditions are as follows: depth $5 \mu \mathrm{m}$ per double stroke, longitudinal feed $12 \mathrm{~m} / \mathrm{min}$.

The results of this experiment and the graphs shown in Fig. 5. The experiment was conducted with a triple repetition. The homogeneity of the dispersion was evaluated by the Cochren criterion.

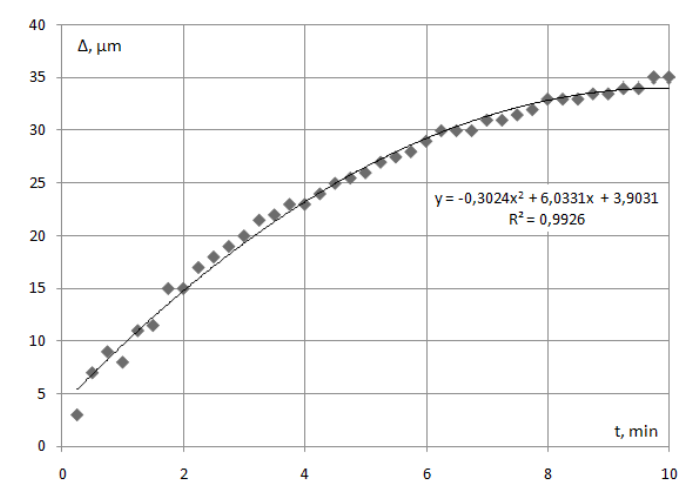

Fig. 5. Plot of dependence wear of grinding wheel on the time

The abscissa axis is the time (t) in minutes; the ordinate axis is the wear $(\Delta)$ of the wheel in micrometers. The resulting graph describes the mode of running-in tool and normal wear, and therefore, the proposed system can be used to assess the state of the grinding wheel during processing.

The condition of the surface of the wheel after processing is shown in Fig. 6. A characteristic change in the state of the circle during the processing of the steel is an intensive self-sharpening with grain chipping, accompanied by local salting.

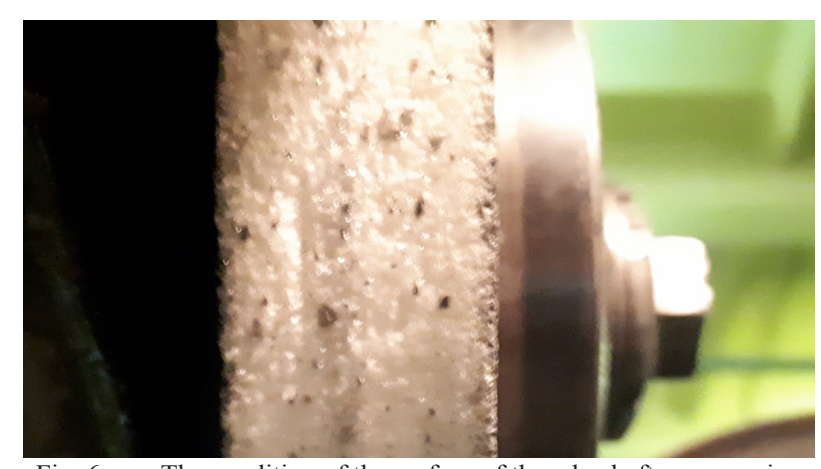

Fig. 6. The condition of the surface of the wheel after processing

\section{Conclusions}

The developed system can be used in mass production to determine the frequency of dressing of the grinding wheel when processing small blanks, and to assess the condition of the wheel and to increase dimensional accuracy when processing large-sized blanks. In a single production, the developed system can be used to assess the state of a circle, determine the processes occurring during grinding and the need for dressing.

\section{REFERENCES}

[1] E. N. Maslov, Theory of grinding materials. Moscow, RU: Machinebuilding, 1974.

[2] A. M. Zahezin and T.V. Maluisheva, Determination of grinding wheel wear by machine vibration parameters. Chelyabinsk, RU: Vestnik YUGU, 2007, №11, pp. 48-53.

[3] K. Furutani, N. Ohguro1 and N. Trong, In-process Measurement of Topography of Grinding Wheel by Using Hydrodynamic Pressure. Nagoya, JP: Toyota Technological Institute.

[4] H. K. Toenshoff, B. Karpuschewski, F. Werner, Fast Sensor System for Diagnosis of Grinding Wheel and Workpiece. Cincinatti, Ohio, USA: 5th Intern. Grinding Conf., 1993.

[5] B. E. Bolotov and V. V. Trubnikov, "Method for contactless measurement of grinding wheel vibrations", SU Patent 444632. September 30, 1975.

[6] A. McDonald, Investigation of Grinding Wheel Wear Using a White Chromatic Sensor, Nova Scotia, CA: Dalhousie University Halifax, 2015. 